\title{
Bounds on the Correlations and Analyticity Properties of Ferromagnetic Ising Spin Systems *
}

\author{
Joel L. Lebowitz \\ Belfer Graduate School of Science, Yeshiva University, New York, N.Y., USA
}

Received July 1, 1972

\begin{abstract}
We consider a ferromagnetic Ising spin system isomorphic to a lattice gas with attractive interactions. Using the Fortuin, Kasteleyn and Ginibre (FKG) inequalities we derive bounds on the decay of correlations between two widely separated sets of particles in terms of the decay of the pair correlation. This leads to bounds on the derivatives of various orders of the free energy with respect to the magnetic field $h$, and reciprocal temperature $\beta$. In particular, if the pair correlation has an upper bound (uniform in the size of the system) which decays exponentially with distance in some neighborhood of $\left(\beta^{\prime}, h^{\prime}\right)$ then the thermodynamic free energy density $\psi(\beta, h)$ and all the correlation functions are infinitely differentiable at $\left(\beta^{\prime}, h^{\prime}\right)$. We then show that when only pair interactions are present it is sufficient to obtain such a bound only at $h=0$ (and only in the infinite volume limit) for systems with suitable boundary conditions. This is the case in the two dimensional square lattice with nearest neighbor interactions for $0 \leqq \beta<\beta_{0}$, where $\beta_{0}^{-1}$ is the Onsager temperature at which $\psi(\beta, h=0)$ has a singularity. For $\beta>\beta_{0}, \partial \psi(\beta, h) / \partial h$ is discontinuous at $h=0$, i.e. $\beta_{0}=\beta_{c}$, where $\beta_{c}^{-1}$ is the temperature below which there is spontaneous magnetization.
\end{abstract}

\section{Introduction}

The concept of a "correlation length", $x^{-1}$, defined so that regions of a system whose spatial separation is greater than $\varkappa^{-1}$ are "essentially" uncorrelated plays an important part in the study of critical point phenomena and generally serves to characterize the degree of order which a system possesses ${ }^{1}$. It is therefore important to understand the relation between the (possibly) different correlation lengths obtained by looking at the decay of correlations between different pairs of observables, "located a distance $r$ apart", as $r \rightarrow \infty,[3,5]$. In this note we prove that for ferromagnetic spin systems the decay of the spin-spin pair correlation "dominates" the decay of all higher order correlations. This result is combined with some observations about the relationship between deriv-

${ }^{\star}$ Research supported by AFOSR Contract \# F 44620-71-C-0013.

1 The concept of a correlation length and its relation to critical point phenomena goes back at least to Ornstein and Zernike [1]. Fisher in particular has emphasized the importance of this quantity [2,3], see in particular [3] for more references. The general relation between clustering and the uniqueness of the equilibrium state is found in Ruelle [4] and references contained there. 
atives of the free energy (and of the different spin expection values) with respect to $\beta$ and $h$ and the decay of correlations to obtain bounds on such derivatives.

The systems we consider are such that their Hamiltonians satisfy the conditions of the Fortuin, Kasteleyn and Ginibre (FKG) inequalities [6]. An important class of such systems (and this note will deal primarily with them) are Ising spin systems with ferromagnetic pair interactions, whose Hamiltonians have the form

$$
H_{\Lambda}=-\frac{1}{2} \sum_{\substack{i, j \in \Lambda \\ i \neq j}} J_{i j} \sigma_{i} \sigma_{j}-h \sum_{i \in \Lambda} \sigma_{i}-\sum_{\substack{i \in \Lambda \\ j \in \tilde{A}}} J_{i j} \sigma_{i} \bar{\sigma}_{j}
$$

Here $\Lambda$ is a finite subset of a $v$-dimensional lattice $Z^{v}, \sigma_{i}= \pm 1$ is an Ising spin variable at each lattice site, $i \in \Lambda$, the $J_{i j}$ are translation invariant ferromagnetic interactions, $J_{i j} \geqq 0, \sum_{j} J_{0 j}=\alpha<\infty, h$ is a uniform external field, and the variables $\bar{\sigma}_{j}$ in $j \in \tilde{\Lambda}=Z^{v} \backslash \Lambda$, are specified as a boundary condition $b_{A}, \bar{\sigma}_{j}=( \pm 1,0)$. The case $\bar{\sigma}_{j}=0$ for all $j$ corresponds to "free boundaries". (We shall also consider "periodic" boundary conditions.)

Let $f\left(\sigma_{i_{1}}, \ldots, \sigma_{i_{l}}\right)$ and $g\left(\sigma_{j_{1}}, \ldots, \sigma_{j_{m}}\right)$ with $i_{k}, j_{k} \in \Lambda$ be monotone functions (say non-decreasing) of their arguments then FKG show that $f$ and $g$ are positively correlated,

where

$$
\langle f g\rangle-\langle f\rangle\langle g\rangle \geqq 0
$$

$$
\langle\cdot\rangle=\sum_{\left\{\sigma_{i}\right\}}\left\{\cdot \exp \left[-\beta H_{A}\right] / Z_{A}\right\}, \quad Z_{A}=\Sigma \exp \left[-\beta H_{A}\right] .
$$

We shall use (1.2) to prove our basic result that the decay of all the correlation functions is dominated by the decay of the pair correlation function. It will be convenient to use, instead of the $\sigma_{j}$, the lattice gas occupation number variables, $\varrho_{i} \equiv \frac{1}{2}\left(\sigma_{i}+1\right)=(0,1), i \in \Lambda$.

Let $A, B$, be sets of lattice sites: $A, B \subset A$. We define for such sets,

$$
S_{A}=\sum_{i \in A} \varrho_{i} ; \quad \varrho_{A}=\prod_{i \in A} \varrho_{i} .
$$

Clearly, $S_{A}, \varrho_{A}$ and $\left(S_{A}-\varrho_{A}\right)$ are non-negative monotone functions of the $\left\{\varrho_{i}\right\}$ and thus also of the $\left\{\sigma_{i}\right\}$. Hence, by (1.2),

and

$$
\left\langle\varrho_{B}\left(S_{A}-\varrho_{A}\right)\right\rangle \geqq\left\langle\varrho_{B}\right\rangle\left\langle S_{A}-\varrho_{A}\right\rangle \geqq 0
$$

$$
\left\langle S_{A}\left(S_{B}-\varrho_{B}\right)\right\rangle \geqq\left\langle S_{A}\right\rangle\left\langle S_{B}-\varrho_{B}\right\rangle \geqq 0
$$

Combining (1.5) and (1.6) yields the desired result;

\section{Lemma 1.}

$$
\begin{aligned}
0 \leqq\left\langle\varrho_{A} \varrho_{B}\right\rangle & =\left\langle\varrho_{A}\right\rangle\left\langle\varrho_{B}\right\rangle \leqq\left\langle S_{A} \varrho_{B}\right\rangle-\left\langle S_{A}\right\rangle\left\langle\varrho_{B}\right\rangle \leqq\left\langle S_{A} S_{B}\right\rangle-\left\langle S_{A}\right\rangle\left\langle S_{B}\right\rangle \\
& =\sum_{i \in A} \sum_{j \in B} u_{2}(i, j)
\end{aligned}
$$


where $u_{2}(i, j)$ is the two particle correlation function (Ursell function)

$$
0 \leqq u_{2}(i, j) \equiv\left\langle\varrho_{i} \varrho_{j}\right\rangle-\left\langle\varrho_{i}\right\rangle\left\langle\varrho_{j}\right\rangle=\frac{1}{4}\left[\left\langle\sigma_{i} \sigma_{j}\right\rangle-\left\langle\varrho_{i}\right\rangle\left\langle\sigma_{j}\right\rangle\right] \leqq \frac{1}{2} .
$$

If $u_{2}(i, j)$ is bounded by a monotone non-increasing function of the Euclidean distance $r_{i j}$ between the $i$-th and $j$-the site, $u_{2}(i, j) \leqq \bar{u}\left(r_{i j}\right)$ then it follows from Lemma 1 that

$$
0 \leqq\left\langle\varrho_{A} \varrho_{B}\right\rangle-\left\langle\varrho_{A}\right\rangle\left\langle\varrho_{B}\right\rangle \leqq|A||B| \bar{u}(d(A, B))
$$

where $|A|,|B|$ are the number of sites in $A$ and $B$ respectively and $d(A, B)$ is the "distance" between the sets $A$ and $B$. (We may choose the nearest neighbor distance as unity.)

The inequalities (1.7)-(1.9) will clearly remain valid also in the thermodynamic limit, $A \rightarrow \infty$. Hence, denoting by $B+\boldsymbol{r}$ the set $B$ translated by a lattice vector $\boldsymbol{r}$, with length $|\boldsymbol{r}|$, the correlations between $\varrho_{A}$ and $\varrho_{B+\boldsymbol{r}}$ will decay asymptotically, $|\boldsymbol{r}| \rightarrow \infty$, at least as fast $\bar{u}(|\boldsymbol{r}|)$.

The spin correlations $\left\langle\sigma_{A} \sigma_{B}\right\rangle-\left\langle\sigma_{A}\right\rangle\left\langle\sigma_{B}\right\rangle$, where $\sigma_{A}=\prod_{i \in A} \sigma_{i}$ can be expressed as sums of terms of the form $\left\langle\varrho_{A^{\prime}} \varrho_{B^{\prime}}\right\rangle-\left\langle\varrho_{A^{\prime}}\right\rangle\left\langle\varrho_{B^{\prime}}\right\rangle$ where $A^{\prime} \subset A$ and $B^{\prime} \subset B$ so that their decay as $d(A, B) \rightarrow \infty$ is also dominated by that of $\bar{u}(d(A, B))$.

We shall also define the higher order Ursell (cluster) functions $u_{i}\left(i_{1}, \ldots, i_{i}\right)$ as $[4,7]$,

$$
\begin{aligned}
u_{3}(i, j, k)= & \left\langle\varrho_{i} \varrho_{j} \varrho_{k}\right\rangle-\left\langle\varrho_{i}\right\rangle\left\langle\varrho_{j} \varrho_{k}\right\rangle-\left\langle\varrho_{j}\right\rangle\left\langle\varrho_{i} \varrho_{k}\right\rangle-\left\langle\varrho_{k}\right\rangle\left\langle\varrho_{i} \varrho_{j}\right\rangle \\
& +2\left\langle\varrho_{i}\right\rangle\left\langle\varrho_{j}\right\rangle\left\langle\varrho_{k}\right\rangle, \ldots
\end{aligned}
$$

The definition of the Ursell functions is chosen so as to make them vanish, for $l \geqq 2$, whenever any subset of the variables in the expectation values is independent of the rest. The corresponding spin Ursell functions, with $\sigma_{i}, \sigma_{j}, \ldots$, replacing $\varrho_{i}, \varrho_{j}, \ldots$, in (1.10) will be denoted by $U_{2}(i, j)$, etc., and it is readily found that

$$
U_{l}\left(i_{1}, \ldots, i_{l}\right) \rightarrow 2^{i} u_{l}\left(i_{1}, \ldots, i_{i}\right) \text { for } l \geqq 2 .
$$

It follows from the definition of the Ursell functions [4, 7], that for any partition of the set $\left\{i_{1}, \ldots, i_{l}\right\}$ into two disjoint sets, $A$ and $B, U_{l}\left(i_{1}, \ldots, i_{l}\right)$ can be written as a finite sum of terms each having the form

$$
( \pm 1) \prod_{\alpha}\left\langle\varrho_{X_{\alpha}}\right\rangle\left[\left\langle\varrho_{A^{\prime} \cup B^{\prime}}\right\rangle-\left\langle\varrho_{A^{\prime}}\right\rangle\left\langle\varrho_{B^{\prime}}\right\rangle\right]
$$

where $A^{\prime} \subset A, B^{\prime} \subset B$ and the disjoint sets $\left\{\left\{X_{\alpha}\right\}, A^{\prime}, B^{\prime}\right\}$ exhaust $\left\{i_{1}, \ldots, i_{l}\right\}$. Hence, using (1.9) and the bound $\left|\varrho_{X}\right|<1$, we find that

$$
\left|U_{i}\left(i_{1}, \ldots, i_{i}\right)\right| \leqq C_{\imath} \bar{u}(d(A, B))
$$

22 Commun. math. Phys., Vol. 28 
with $C_{l}$ some finite constant. Consequently, we always have,

$$
\left|U_{l}\left(i_{1}, \ldots, i_{l}\right)\right| \leqq C_{l} \bar{u}\left(R_{i} / l\right),
$$

where $R_{i}$ is the "diameter" of the set $\left\{i_{1}, \ldots, i_{i}\right\}, R_{l}=\operatorname{Max}\left\{\left|\boldsymbol{r}_{i_{j}}-\boldsymbol{r}_{i_{k}}\right|\right\}$, $1 \leqq j, k \leqq l$.

\section{Differentiability Properties in the Thermodynamic Limit}

The free energy density per unit volume (multiplied by $-\beta$ ) of the system specified by the Hamiltonian (1.1) is defined as

$$
\psi\left(\beta, \tilde{h} ; \Lambda, b_{\Lambda}\right)=|\Lambda|^{-1} \ln Z_{\Lambda}
$$

where we have set $\beta h=\tilde{h},|\Lambda|$ is the number of sites in $\Lambda$, and $b_{A}$ indicates the boundary conditions. The following statements are known to be true for the thermodynamic limit, $\Lambda \rightarrow \infty$, of the free energy density

$$
\psi(\beta, \tilde{h})=\lim _{\Lambda \rightarrow \infty} \psi\left(\beta, \tilde{h} ; \Lambda, b_{\Lambda}\right),
$$

and of the correlation functions

for references see $[4,7,9]$.

$$
\left\langle\sigma_{A}\right\rangle(\beta, \tilde{h})=\lim _{\Lambda \rightarrow \infty}\left\langle\sigma_{A}\right\rangle\left(\beta, \tilde{h} ; \Lambda, b_{\Lambda}\right) ;
$$

(i) $\psi(\beta, \tilde{h})$ exists (independent of $\left.b_{A}\right)$ and is continuous in $\beta$ and $h$ for all real $\beta$ and $\tilde{h}$.

(ii) $\psi$ is analytic in the complex $\tilde{h}$-plane for $\operatorname{Re} \tilde{h} \neq 0$ and $\beta \geqq 0$.

(iii) $\psi$ is real analytic in $\beta$ for $\beta \geqq 0$ when $\operatorname{Re} \tilde{h} \neq 0$.

(iv) There exists a $\beta^{\prime}>0$ such that for $\beta \leqq \beta^{\prime}, \psi(\beta, \tilde{h})$ is also real analytic in $\beta$ and $\tilde{h}$ at $\tilde{h}=0$.

(v) The thermodynamic limit of the correlation functions $\left\langle\sigma_{A}\right\rangle(\beta, \tilde{h})$, exist (independent of $b_{A}$ ), are translation invariant, having some clustering property and are continuous in $\tilde{h}$ at all those values of $\beta \geqq 0$, and of $\tilde{h}$ at which $\psi(\beta, \tilde{h})$ is differentiable with respect to $\tilde{h},[10]$.

(vi) The regions of $\beta$ and $\tilde{h}$, mentioned in (ii)-(iv), at which $\psi$ is known to be analytic are also regions of analyticity for the $\left\langle\sigma_{A}\right\rangle(\beta, \tilde{h})$ with the same analyticity properties as $\psi(\beta, \tilde{h})$.

Thus, if we define the reciprocal critical temperature $\beta_{c}$ as the value of $\beta$ above which the spontanuous magnetization, which is a nondecreasing function of $\beta$, is positive;

$$
m^{*}(\beta) \equiv \lim _{\tilde{h} \rightarrow 0+} m(\beta, \tilde{h})=\left\{\begin{array}{r}
0, \beta<\beta_{c} \\
>0, \beta>\beta_{c}
\end{array}\right.
$$

where

$$
m(\beta, \tilde{h}) \equiv \partial \psi(\beta, \tilde{h}) / \partial \tilde{h},
$$


we have, at $\tilde{h}=0$, uniqueness and continuity of the correlation functions and differentiability of $\psi(\beta, \tilde{h})$ (in $\tilde{h}$ ) for $\beta<\beta_{c}$ and analyticity in $\tilde{h}$ and $\operatorname{Re} \beta$ for $\beta \leqq \beta^{\prime}<\beta_{c}$ (for $\tilde{h} \neq 0$ the analyticity holds of course for all $\beta$ ). For $\beta>\beta_{c}$ the correlations $\left\langle\sigma_{A}\right\rangle(\beta, \tilde{h})$ are discontinuous at $\tilde{h}=0$ and the limit $\Lambda \rightarrow \infty$ of $\left\langle\sigma_{A}\right\rangle\left(\beta, \tilde{h}=0 ; \Lambda, b_{A}\right)$ will depend on the boundary conditions $b_{A}[10]$.

There are still many questions, however about the analytic nature of $\psi(\beta, \tilde{h})$ and of the $\left\langle\sigma_{A}\right\rangle$ at $\tilde{h}=0$ and $\beta^{\prime}<\beta<\beta_{c}$.

We shall now show that we may use (1.7) to establish such results for $\psi(\beta, \tilde{h})$ and $\left\langle\sigma_{\boldsymbol{A}}\right\rangle(\beta, \tilde{h})$ when something is known about the asymptotic behavior of the two-particle Ursell function in the neighborhood of $\tilde{h}=0$. (It is known that when the pair potential $J_{i j}$ has a finite range, $J_{i j}=0$ for $r_{i j}>R$, (or decays exponentially) then the correlations

$$
\left[\left\langle\sigma_{A} \sigma_{B}\right\rangle-\left\langle\sigma_{A}\right\rangle\left\langle\sigma_{B}\right\rangle\right],
$$

and also all Ursell functions, decay exponentially fast for any fixed $\tilde{h} \neq 0$ or when $\beta \leqq \beta^{\prime}$, [7].) To this end we shall make use of the following relationship, between the derivatives of the free energy and sums over the $U_{l}$,

$$
\begin{aligned}
& \frac{\partial}{\partial \tilde{h}}\left\langle\sigma_{A}\right\rangle\left(\beta, \tilde{h} ; \Lambda, b_{A}\right) \\
& \quad=\sum_{i \in \Lambda}\left[\left\langle\sigma_{A} \sigma_{i}\right\rangle\left(\beta, \tilde{h} ; \Lambda, b_{\Lambda}\right)-\left\langle\sigma_{A}\right\rangle\left(\beta, \tilde{h} ; \Lambda, b_{\Lambda}\right)\left\langle\sigma_{i}\right\rangle\left(\beta, \tilde{h} ; \Lambda, b_{\Lambda}\right)\right], \\
& \frac{\partial^{m}}{\partial \tilde{h}^{m}}\left\langle\sigma_{i}\right\rangle\left(\beta, \tilde{h} ; \Lambda, b_{\Lambda}\right) \\
& \quad=\sum_{j_{1}, \ldots, j_{m} \in \Lambda} U_{m+1}\left(i, j_{1}, \ldots, j_{m}, \beta, \tilde{h} ; \Lambda, b_{\Lambda}\right), \quad m=1,2, \ldots, \\
& \frac{\partial \psi\left(\beta, \tilde{h} ; \Lambda, b_{\Lambda}\right)}{\partial \tilde{h}}=\frac{1}{|\Lambda|} \sum_{i \in \Lambda}\left\langle\sigma_{i}\right\rangle\left(\beta, \tilde{h} ; \Lambda, b_{\Lambda}\right) \equiv m\left(\beta, \tilde{h} ; \Lambda, b_{\Lambda}\right) .
\end{aligned}
$$

Eqs. (2.4)-(2.5) can be readily obtained by explicit computation $[4,7,9]$. Expressions similar to (2.4)-(2.5) (involving sums of combinations of different order Ursell functions) hold for the derivatives of $\psi$ and $\left\langle\sigma_{A}\right\rangle$ with respect to $\beta$, as well as for mixed derivatives.

It is now readily seen that when the magnitude of each term in the sum on the right side of (2.4) is bounded in the neighborhood of $\tilde{h}=0$, uniformly in $\Lambda$, for all $m<k, k \geqq 1$, and the sum of the bounds converges, then $\psi(\beta, \tilde{h})$ is $k$ times differentiable at $\tilde{h}=0$. In particular if, for some boundary condition $b_{\Lambda}, U_{2}\left(i, j, \beta, \tilde{h} ; \Lambda, b_{\Lambda}\right) \leqq K r_{i j}^{-(v+\varepsilon)}$, with $K$ and $\varepsilon$ fixed positive constants independent of $\Lambda$ and $\tilde{h}$ then taking the thermo- 
dynamic limit (via some subsequence, if necessary) of $\left\langle\sigma_{A}\right\rangle\left(\beta, \tilde{h} ; \Lambda, b_{A}\right)$ we find that the limit $\left\langle\sigma_{A}\right\rangle(\beta, \tilde{h})$ is continuous and hence independent of $b_{A}$, even at $\tilde{h}=0$, since it is independent of $b_{A}$ for $\tilde{h} \neq 0$. Consequently, since each term on the right side of $(2.4 \mathrm{a})$ is continuous in $\tilde{h}$ and the series converges uniformly, $\partial\left\langle\sigma_{A}\right\rangle(\beta, \tilde{h}) / \partial \tilde{h}$ is continuous. The magnetization $m(\beta, \tilde{h})$, an odd function of $\tilde{h}$, has a continuous derivative at $\tilde{h}=0$ and $\psi(\beta, \tilde{h})$ is twice differentiable. Similar results hold for differentiation with respect to $\beta$ (and the cross derivative of $\psi(\beta, \tilde{h})$ ) in any open interval, $\beta_{1}<\beta<\beta_{2}$, in which the bound holds uniformly.

More generally a uniform bound on $U_{2}$ of the form $\bar{u}(r)=K r^{-(k v+\varepsilon)}$ will make $\psi(\beta, \tilde{h}) \in C^{k+1}$ and $\left\langle\sigma_{A}\right\rangle \in C^{k}$ in both variables. Finally if the pair correlation decays exponentially

$$
U_{2}\left(i, j, \beta, \tilde{h} ; \Lambda, b_{\Lambda}\right) \leqq K \exp \left[-\varkappa r_{i j}\right],
$$

with $K$ and $\varkappa>0$, independent of $\Lambda$ and $\tilde{h}$ (around $\tilde{h}=0)$ then $\psi(\beta, \tilde{h})$ and $\left\langle\sigma_{A}\right\rangle(\beta, \tilde{h}) \in C^{\infty}$ in $\tilde{h}$, (and also with respect to $\beta$ in an open $\beta$-interval in which the bound (2.6) held uniformly). This can be seen by using (1.13) in (2.4) to give,

$$
\left|\frac{\partial^{m}}{\partial \tilde{h}^{m}}\left\langle\sigma_{i}\right\rangle\left(\beta, \tilde{h} ; \Lambda, b_{\Lambda}\right)\right| \leqq D_{m} \sum_{\boldsymbol{r}} \bar{u}(r) r^{(m-1) v}
$$

where $D_{m}$ is a finite constant and the sum is over all lattice sites with the site $i$ serving as the origin. (Unfortunately, as $k \rightarrow \infty, D_{k}$ grows as $b^{k} C_{k} k^{v k}$, where $b$ is a constant and $C_{k}$ is defined in (1.12).) $C_{k}$ itself may grow as $k$ ! so that we cannot, with the present bounds, establish analyticity at $\tilde{h}=0$ even when (2.6) holds.

\section{Bounds at $\tilde{h}=0$ and the Two Dimensional Ising System}

In order to apply the results of the last sections to obtain information about the behavior of $\psi$ and $\left\langle\sigma_{A}\right\rangle$ at a particular value of $\beta$ and $\tilde{\mathrm{h}}$ we need (for a general system satisfying the FKG inequalities) to have a bound on $U_{2}\left(i, j \beta, \tilde{h} ; \Lambda, b_{A}\right)$, in a neighborhood of $(\beta, \tilde{h})$, which is uniform in $\Lambda$. We shall show however that for the Ising spin system with only ferromagnetic pair interactions (the system we have been considering explicitely) it is sufficient to have a bound valid in the thermodynamic limit (for boundary conditions to be specified) just at $\tilde{h}=0$ and some value $\bar{\beta}$ to deduce the desired results for all values of $\tilde{h}$ and all $0 \leqq \beta \leqq \bar{\beta}$. To this end we establish the following lemma.

Lemma 2. For an Ising spin system with only ferromagnetic pair interactions,

$$
\begin{aligned}
U_{2}\left(i, j, \beta, \tilde{h} ; \Lambda, b_{0}\right) & \leqq U_{2}\left(i, j, \beta, 0 ; \Lambda, b_{0}\right) \leqq U_{2}\left(i, j, \bar{\beta}, 0 ; \bar{\Lambda}, b_{0}\right) \\
& \leqq U_{2}\left(i, j, \bar{\beta}, 0 ; \bar{\Lambda}, b_{p}\right)
\end{aligned}
$$


for $\beta \leqq \bar{\beta}$ and $\Lambda \subset \bar{\Lambda}$. Here $b_{0}$ indicates free boundaries, $\bar{\sigma}_{j}=0$ in (1.1), and $b_{p}$ indicates "periodic" boundary conditions. (This includes cylindrical boundary conditions which are periodic in some directions and free in others. "Screw" boundary conditions are also included. For all these boundary conditions $\boldsymbol{r}_{i j}$ is defined with the proper "modulo".)

Proof. The first inequality in (3.1) follows from the work of Griffiths, Hurst and Sherman [11] who proved that $U_{3}\left(i, j, k, \beta, \tilde{h} ; \Lambda, b_{0}\right)$, which is an odd function of $\tilde{h}$, is non-positive for $\tilde{h} \geqq 0$. But, as seen from (2.4),

$$
\frac{\partial U_{2}\left(i, j, \beta, \tilde{h} ; \Lambda, b_{0}\right)}{\partial \tilde{h}}=\sum_{k \in \Lambda} U_{3}\left(i, j, k, \beta, \tilde{h} ; \Lambda, b_{0}\right) .
$$

Hence, $U_{2}\left(i, j, \beta, \tilde{h} ; \Lambda, b_{0}\right)$, which is a non-negative even function of $\tilde{h}$, has its maximum value at $\tilde{h}=0$. (The same is true for periodic boundary conditions.)

To obtain the second inequality in (3.1) we note that $U_{2}\left(i, j, \beta, 0 ; \Lambda, b_{0}\right)$ $=\left\langle\sigma_{i} \sigma_{j}\right\rangle\left(\beta, 0 ; \Lambda, b_{0}\right)$, since $\left\langle\sigma_{k}\right\rangle\left(\beta, 0 ; \Lambda, b_{0}\right)=0$ by symmetry. We then use the result of Griffiths [9] that $\left\langle\sigma_{i} \sigma_{j}\right\rangle\left(\beta, \tilde{h} ; \Lambda, b_{0}\right)$ is monotone increasing in $\beta$ and in the "size" of $\Lambda$; since increasing $\beta$ or $\Lambda$ increases the ferromagnetic interactions. The last inequality holds for the same reason; there are more ferromagnetic interactions in the periodic case.

It follows from (3.1) that (2.6), with $b_{\Lambda}=b_{0}$, will hold for all values of $\tilde{h}$ and all $\beta^{\prime} \leqq \beta$ whenever

$$
\lim _{\Lambda \rightarrow \infty}\left\langle\sigma_{i} \sigma_{j}\right\rangle\left(\beta, \tilde{h}=0 ; \Lambda, b_{p}\right) \leqq K \exp \left[-x r_{i j}\right], \quad K<\infty, x>0 .
$$

An inequality of the form (3.3) can be established for the two dimensional square lattice with nearest neighbor interactions for $\beta<\beta_{0} ; \beta_{0}$ is the (reciprocal) Onsager temperature defined by the relation $\sinh \left(2 \beta_{0} J_{1}\right)$ $\sinh \left(2 \beta_{0} J_{2}\right)=1$, with $J_{1}$ and $J_{2}$ the "horizontal" and "vertical" nearest neighbor interaction. The value of $x$ in (3.3) is essentially equal to $1 n(1+\Delta)$ with $\Delta$ proportional to the "gap" in the spectrum of the transfer matrix which is positve, $\Delta>0$ for $\beta<\beta_{0}$ [12].

The bound (3.3) is a direct consequence of the expression for $\left\langle\sigma_{i} \sigma_{j}\right\rangle$ in terms of the eigenvalues and eigenvectors of the transfer matrix $[3,12,13]$. With cylindrical boundary conditions and $i$ and $j$ on the same "row" (along the cylinder axis) the result is immediate, for "arbitrary" directions use has to be made of the "symmetry" of the eigenvectors of the transfer matrix (M. E. Fisher, private communication). Using more abstract arguments Marinaro and Sewell [14] have shown recently that a bound of the form (3.3) will hold for all correlations

$$
\left|\left\langle\sigma_{A} \sigma_{B+r}\right\rangle-\left\langle\sigma_{A}\right\rangle\left\langle\sigma_{B+r}\right\rangle\right|
$$


for an Ising system with arbitrary finite range interactions whenever there is a gap in the spectrum of the transfer matrix.

We have thus established that for this two dimensional system $\psi(\beta, \tilde{h})$ and $\left\langle\sigma_{A}\right\rangle(\beta, \tilde{h})$ are $C^{\infty}$ in $\tilde{h}$ for $\beta<\beta_{0} \leqq \beta_{c}$. We also know [15], that the formula for the "long range order spontaneous magnetization", $m^{*}(\beta)$ $=\left[1-\left(\sin h \beta / \beta_{0}\right)^{-4}\right]^{1 / 8}$ for $\beta \geqq \beta_{0}$, calculated by Onsager and Yang, is a lower bound for $m^{*}(\beta)$, i.e. $m^{*}(\beta)>0$ for $\beta>\beta_{0}$ which implies $\beta_{0} \geqq \beta_{c}$. Hence $\beta_{0}=\beta_{c}$ for this system.

For more general Ising spin systems with ferromagnetic pair interactions Fisher [16] has obtained a bound on $\left\langle\sigma_{i} \sigma_{j}\right\rangle(\beta, \tilde{h}=0)$ in terms of sums over self-avoiding walks on the lattice. These bounds which Fisher has used to obtain lower bounds on $\beta_{c}$, may be combined with our results to obtain information also about the derivatives of $\psi$ and $\left\langle\sigma_{A}\right\rangle$.

Concluding Remarks. (i) The above results (which do not involve explicitely the Onsager result) apply also to higher spin Ising systems, i.e. $\sigma_{i}=l, l-2, \ldots,-l, l>1$, with ferromagnetic pair interactions [9].

(ii) The following may be useful for Ising systems with ferromagnetic pair interactions at $\beta>\beta_{c}$. Let $\tilde{h}_{\Lambda}^{\prime} \geqq 0$ be a field, which depends on $\Lambda$, with $\tilde{h}_{\Lambda}^{\prime} \rightarrow 0$ as $\Lambda \rightarrow \infty$. Then, by [11], for $\tilde{h}>0$,

Hence

$$
U_{2}\left(i, j, \beta, \tilde{h}+\tilde{h}_{\Lambda}^{\prime} ; \Lambda, b_{0}\right) \leqq U_{2}\left(i, j, \beta, \tilde{h}_{\Lambda} ; \Lambda, b_{0}\right) .
$$

$$
U_{2}(i, j, \beta, \tilde{h}) \leqq U_{2}(i, j, \beta, 0+) \leqq \tilde{U}_{2}(i, j, \beta) \equiv \liminf _{\Lambda \rightarrow \infty} \tilde{U}_{2}\left(i, j, \beta, \tilde{h}_{\Lambda} ; \Lambda, b_{0}\right) .
$$

Thus if we have an exponential bound on $\tilde{U}_{2}(i, j, \beta),\left\langle\sigma_{A}\right\rangle(\beta, \tilde{h})$ will be $C^{\infty}$ as $\tilde{h} \rightarrow 0$. When this is true in some neighborhood of $\beta$ then $m^{*}(\beta)$ is also $C^{\infty}$ at $\beta$.

Acknowledgements. I am greatly indebted to Michael E. Fisher, Jean Ginibre, Robert B. Griffiths and Elliott $\mathrm{H}$. Lieb for many helpful comments.

\section{References}

1. Ornstein, L. S., Zernike, F.: Z. Physik 19, 134 (1918); 27, 761 (1926).

2. Fisher, M.E.: J. Math. Phys. 5, 944 (1964) [see especially footnote 59].

3. Camp, W. J., Fisher, M.E.: Phys. Rev. B 6, 946 (1972); W.J.Camp, The Decay of order in Many-Body systems. II. The ising model at high temperatures. Phys. Rev. B 6, 960 (1972).

4. Ruelle,D.: Statistical mechanics. New York: Benjamin 1969.

5. Jones, G.L., Coletta, V.P.: Phys. Rev. 171, 428 (1969). - Camp, W. J., Fisher, M.E.: Phys. Rev. Letters 26, 73 (1971). - Fisher, M.E., Camp, W. J.: Phys. Rev. Letters 26, 565 (1971).

6. Fortuin, C.M., Kaseleyn, P.W., Ginibre, J.: Commun. math. Phys. 22, 89 (1971).

7. Lebowitz, J.L., Penrose, O.: Commun. math. Phys. 11, 99 (1968).

8. Lebowitz,J.L.: Ann. Rev. Phys. Chem. 19, 194 (1968). 
9. Griffiths, R.B.: Rigorous results and theorems, to appear in Phase Transitions and Critical Points, edited by C. Domb and M. S. Green. New York: Academic Press 1972.

10. Lebowitz, J. L., Martin-Löf, A.: Commun. math. Phys. 25, 276 (1972).

11. Griffiths, R. B., Hurst, C. A., Sherman, S.: J. Math. Phys. 11, 790 (1970).

12. Onsager, L.: Phys. Rev. 65, 117 (1944).

13. Kaufman, B., Onsager,L.: Phys. Rev. 76, 1244 (1949); cf. also, Montroll,E.W., Potts, R. B., Ward, J.C.: J. Math. Phys. 4, 308 (1963). - Schultz, T.D., Mattis, D. C., Lieb, E.: Rev. Mod. Phys. 36, 856 (1964).

14. Marinaro, M., Sewell, G.L.: Commun. math. Phys. 24, 310 (1972); see also Minlos, R. A., Sinai, Ja. G.: Teor. Matem. Fizika 2 (2), 230 (1970).

15. Griffiths, R.: Phys. Rev. 152, 240 (1966). - Martin-Löf,A.: On the spontaneous magnetization in the Ising Model, preprint.

16. Fisher, M.E.: Phys. Rev. 162, 475 (1967).

Joel L. Lebowitz

Belfer Graduate School of Science Yeshiva University

New York, N.Y. 10033, USA 
